Weber - Einführung in die Betriebswirtschaftslehre 
Wolfgang Weber

\section{Einführung in die}

Betriebswirtschaftslehre

2., aktualisierte und überarbeitete Auflage

\section{GABLER}


Prof. Dr. Wolfgang Weber lehrt Betriebswirtschaftslehre, insbesondere Personalwirtschaft, an der Universität Paderborn.

Die Deutsche Bibliothek - CIP-Einheitsaufnahme

Weber, Wolfgang:

Einführung in die Betriebswirtschaftslehre / Wolfgang Weber. -

2., aktualisierte und überarb. Aufl. - Wiesbaden : Gabler, 1993

(Gabler-Lehrbuch)

1. Auflage 1991

Nachdruck 1992

2. Auflage 1993

(C) Springer Fachmedien Wiesbaden 1993

Ursprünglich erschienen bei Betriebswirtschaftlicher Verlag Dr . Th. Gabler GmbH, Wiesbaden 1993.

Lektorat: Silke Specht

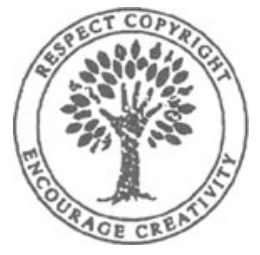

Das Werk einschließlich aller seiner Teile ist urheberrechtlich geschützt. Jede Verwertung außerhalb der engen Grenzen des Urheberrechtsgesetzes ist ohne Zustimmung des Verlages unzulässig und strafbar. Das gilt inshesondere für Vervielfältigungen, Übersetzungen, Mikroverfilmungen und die Einspeicherung und Verarbeitung in elektronischen Systemen.

Höchste inhaltliche und technische Qualität unserer Produkte ist unser Ziel. Bei der Produktion und Auslieferung unserer Bücher wollen wir die Umwelt schonen: Dieses Buch ist auf säurefreiem und chlorfrei gebleichtem Papier gedruckt.

Die Wiedergabe von Gebrauchsnamen, Handelsnamen, Warenbezeichnungen usw. in diesem Werk berechtigt auch ohne besondere Kennzeichnung nicht zu der Annahme, daß solche Namen im Sinne der Warenzeichen- und Markenschutz-Gesetzgebung als frei zu betrachten wären und daher von jedermann benutzt werden dürften. 


\section{Vorwort zur 2. Auflage}

Die erste Auflage dieser Einführung in die Betriebswirtschaftslehre war nach kurzer Zeit vergriffen. 1992 erfolgte zunächst ein Nachdruck. In der Zwischenzeit wurde der Text noch einmal überarbeitet: Es wurden einige zusätzliche Abbildungen zur Veranschaulichung des Textes aufgenommen, der Text an einigen Stellen gestrafft und an anderen erweitert, die Zahlenangaben aktualisiert und - wo immer möglich - auf die gesamte Bundesrepublik Deutschland einschließlich der neuen Bundesländer bezogen. Die offenbar unvermeidlichen Druckfehler wurden hoffentlich alle korrigiert.

Für zahlreiche Anregungen habe ich vielen Kolleginnen und Kollegen zu danken. Bei der Neubearbeitung haben mich Dipl.-Kauffrau Susanne Schmidtmeier und Rüdiger Kabst besonders tatkräftig unterstützt. Auch ihnen danke ich für ihre Unterstützung.

Paderborn

Wolfgang Weber

\section{Vorwort zur 1. Auflage}

Dieses Buch soll in die Betriebswirtschaftslehre einführen. Das bedeutet: Es soll möglich sein, das Buch ohne Vorkenntnisse zu lesen und zu verstehen. Ziel des Autors ist es, daß die Leserinnen und Leser nach der Lektüre mit den wichtigsten Fragen des Faches sowie mit ersten Antworten vertraut sind.

Zielgruppe dieses Buches sind die Interessenten an betriebswirtschaftlichem Grundwissen, vor allem Studierende, aber auch Praktiker, die einen kompakten Gesamtüberblick über die Betriebswirtschaftslehre gewinnen wollen.

Hinter dem Konzept dieses Buches steht die Überlegung, daß die Auseinandersetzung mit einem komplexen Fachgebiet wie dem der Betriebswirtschaftslehre in mehreren Schritten erfolgen muß: einer ersten Kontaktaufnahme mit den Problemen des Faches, einem tieferen Eindringen in die Theo- 
rien bzw. Zusammenhänge betrieblichen Geschehens, um auf dieser Grundlage eigenständige Lösungen betriebswirtschaftlicher Probleme entwickeln zu können. Dieses Buch soll bei dem ersten Schritt behilflich sein. Es kann also die zum Teil hervorragenden Gesamtdarstellungen, meist als Allgemeine Betriebswirtschaftslehre bezeichnet, nicht ersetzen, wohl aber ihren Gebrauch vorbereiten und vielleicht sogar für die tiefer in die Materie eindringenden Studierende ertragreicher gestalten.

An dieser Zielsetzung orientiert sich der Aufbau und die Sprache des Buches. Der Text soll an die nächsten Schritte eines betriebswirtschaftlichen Studiums heranführen. Er soll aber auch als Lehrbuch für solche Interessenten bzw. Studierende dienen können, die aus anderen Disziplinen - z.B. aus den Ingenieurwissenschaften oder aus den Geisteswissenschaften - einen vielleicht nur kurzen Blick auf die Betriebswirtschaftslehre werfen wollen.

$\mathrm{Zu}$ diesem Buch hat mich mein langjähriger Kontakt mit Studienanfängern, mit interessierten Praktikern, aber auch mit Experten für Spezialgebiete, die an einem Gesamtüberblick über die Betriebswirtschaftslehre interessiert sind, ermutigt.

Bei dem Zustandekommen dieses Textes haben mir so viele freundliche und hilfsbereite Menschen geholfen, daß ich gar nicht den Versuch unternehme, sie hier namentlich zu nennen. Vor allem meine Kollegen an der Universität Paderborn und an anderen Hochschulen, mit denen ich an ähnlichen Aufgaben - z.B. bei der Konzipierung und Realisation einer vom Westdeutschen Rundfunk produzierten Fernsehreihe - zusammenarbeiten durfte, gaben mir viele wertvolle Hinweise. In einzelnen Fällen war diese Zusammenarbeit so intensiv, daß ich heute - wie etwa beim Kapitel Rechnungswesen - nicht mehr weiß, ob ein bestimmter Gedanke von mir oder von einem Kollegen - hier von Otto Rosenberg - stammt. All meinen Kollegen sowie meinen Mitarbeiterinnen und Mitarbeitern, die mich bei der technischen Umsetzung in ein druckreifes Manuskript unterstützten, danke ich für ihre Hilfe. 


\section{Inhaltsverzeichnis}

Teil 1: Grundlagen und Rahmenbedingungen $\ldots \ldots \ldots \ldots \ldots$

1. Gegenstand der Betriebswirtschaftslehre . . . . . . . . . . 1

1.1 Betriebswirtschaftslehre als Teil der

Wirtschaftswissenschaften . . . . . . . . . . . . .

1.2 Betriebswirtschaftslehre im System der Wissenschaften . . . . . .3

1.3 Betriebswirtschaftliche Funktionen . . . . . . . . . . 5

1.3.1 Die leistungswirtschaftlichen Funktionen

Beschaffung, Produktion und Absatz . . . . . . . . 7

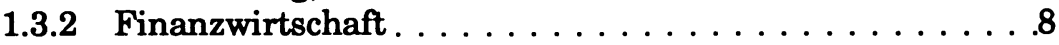

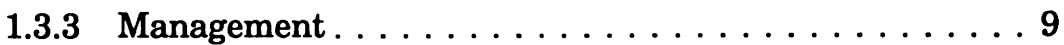

1.4 Betriebstypen . . . . . . . . . . . . . . . . 10

1.5 Teilgebiete der Betriebswirtschaftslehre . . . . . . . . . . .14

2. Gesellschaftliches, wirtschaftliches und rechtliches Umfeld . . . . . . 18

2.1 Gesellschaftliches Umfeld . . . . . . . . . . . . . . . 18

2.2 Wirtschaftliches Umfeld . . . . . . . . . . . . . . 20

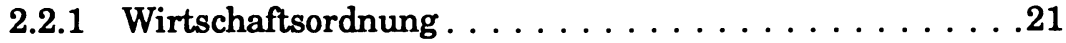

2.2.2 Wirtschaftliche Entwicklung . . . . . . . . . . .23

2.2.3 Steuersystem . . . . . . . . . . . . . . . 24

2.2.4 Wahl des wirtschaftlichen Umfeldes: Die

Standortentscheidung . . . . . . . . . . 25

2.3 Rechtliches Umfeld: Unternehmensverfassung . . . . . . . . . 27

2.3.1 Begriff und Inhalt der Unternehmensverfassung . . . . . .27

2.3.2 Rechtsformen . . . . . . . . . . . . . . 29

2.3.2.1 Übersicht . . . . . . . . . . . . . . 29

2.3.2.2 Kurzcharakterisierung der wichtigsten Rechtsformen

des Privatrechts . . . . . . . . . . . . . 30

2.3.2.3 Kriterien für die Wahl der Rechtsform . . . . . . . 34

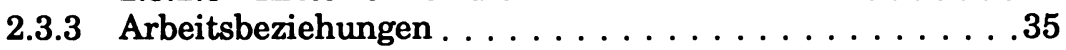

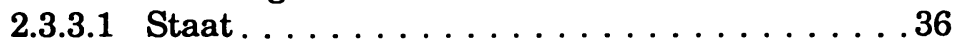

2.3.3.2 Tarifautonomie . . . . . . . . . . . . . 37

2.3.3.3 Unternehmensebene . . . . . . . . . . . . . . . 39

2.3.3.4 Betriebsebene . . . . . . . . . . . . . . . . 40

2.3.3.5 Individueller Arbeitsvertrag . . . . . . . . . 40

2.3.4 Grundzüge der Mitbestimmung . . . . . . . . . . .40

2.3.4.1 Mitbestimmung auf Unternehmensebene . . . . 40

2.3.4.2 Mitbestimmung auf Betriebsebene . . . . . . . .43 


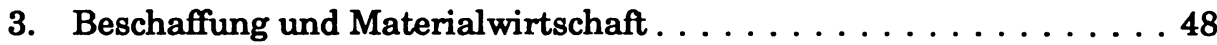

3.1 Grundlagen . . . . . . . . . . . . . . . . . 48

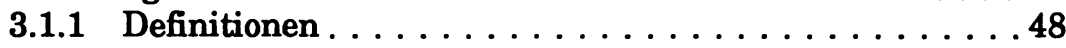

3.1.2 Beschaffungsziele . . . . . . . . . . . . . 49

3.1.3 Bedeutung der Materialwirtschaft . . . . . . . . . 51

3.2 Grundsatzentscheidungen . . . . . . . . . . . . 52

3.3 Gestaltung des Beschaffungsvorgangs . . . . . . . . . 54

3.3.1 Zusammenhänge zwischen Beschaffungszielen und

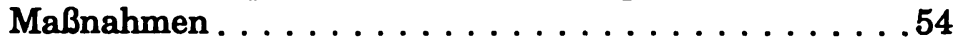

3.3.2 Beschaffungsplanung . . . . . . . . . . . . . . .55

3.3.3 Durchführung des Beschaffungsvorgangs . . . . . . .60

3.4 Lagerhaltung . . . . . . . . . . . . . . . . . . . .60

3.5 Umweltorientierte Materialwirtschaft und Entsorgung . . . . . .62

3.6 Theoretische Grundlagen . . . . . . . . . . . . . .63

4. Produktionswirtschaft .................65

4.1 Grundlagen . . . . . . . . . . . . . . . . . .65

4.1.1 Abgrenzungen: Produktion und Produktionsfaktoren . . .65

4.1.2 Produktionsziele . . . . . . . . . . . . . . .666

4.2 Gestaltung der Rahmenbedingungen . . . . . . . . . . 68

4.2.1 Standort . . . . . . . . . . . . . . . .68

4.2.1.1 Betrieblicher Standort . . . . . . . . . . 668

4.2.1.2 Innerbetrieblicher Standort . . . . . . . . 69

4.2.2 Fertigungstypen . . . . . . . . . . . . .69

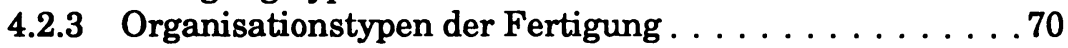

4.3 Produktionsgestaltung . . . . . . . . . . . . . . .72

4.3.1 Gestaltungsfelder . . . . . . . . . . . . . 72

4.3.2 Computerunterstützung der Produktion . . . . . . . . .75

4.3.3 Umweltgerechte Produktion . . . . . . . . . . . . 78

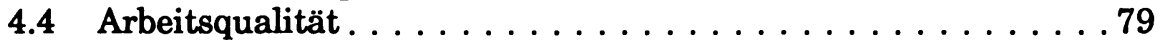

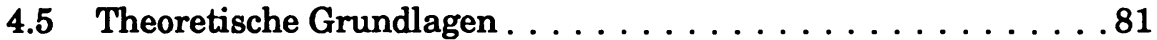

5. Absatzwirtschaft . . . . . . . . . . . . . . . .84

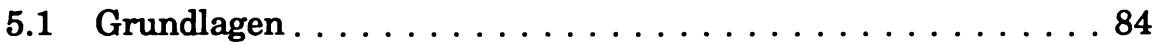

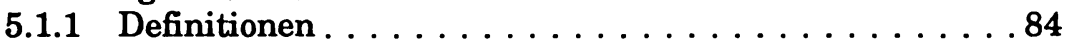

5.1 .2 Bedeutung . . . . . . . . . . . . . . 85

5.2 Gestaltung: Der absatzwirtschaftliche Prozeß . . . . . . . . . 87

5.2.1 Analyse der Absatzchancen . . . . . . . . . . . . .87

5.2.2 Formulierung von Absatzzielen . . . . . . . . . . . 89

5.2.3 Absatzwirtschaftliche Maßnahmen und

Marketing-Mix . . . . . . . . . . . . . . . . . 89

5.2.3.1 Produkt-Mix . . . . . . . . . . . . . .89

5.2.3.2 Kontrahierungs-Mix . . . . . . . . . . . . .92

5.2.3.3 Distributions-Mix . . . . . . . . . . . . .93

5.2.3.4 Kommunikations-Mix . . . . . . . . . . .95

5.2.3.5 Marketing-Mix . . . . . . . . . . . .98 
5.2.4 Durchführung und Kontrolle der absatzwirtschaftlichen

Maßnahmen . . . . . . . . . . . . . . . . 999

5.3 Verbraucherschutz . . . . . . . . . . . . . . . . 99

5.4 Theoretische Grundlagen . . . . . . . . . . . . . . . 101

6. Finanzwirtschaft . . . . . . . . . . . . . . . . . . . 104

6.1 Grundlagen . . . . . . . . . . . . . . . . . . . . 104

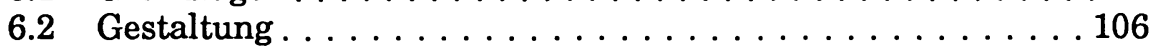

6.2.1 Kapitalbedarfsermittlung . . . . . . . . . . . . 106

6.2.2 Langfristige Finanzierung . . . . . . . . . . . . . . . 109

6.2.2.1 Die Beeinflussung von Kapitalbindung und

Kapitalfreisetzung . . . . . . . . . . . 109

6.2.2.2 Kapitalzuführung . . . . . . . . . . . . 110

6.2.2.3 Halten von Liquiditätsreserven . . . . . . . . . 112

6.2.3 Kurzfristige Finanzierung . . . . . . . . . . . . . . . .113

6.2.4 Strukturelle Liquidität . . . . . . . . . . . . . . . . . . 114

6.3 Finanzinstitutionen . . . . . . . . . . . . . . . . 117

6.4 Theoretische Grundlagen . . . . . . . . . . . . . . . . 119

Teil 3: Management $\ldots \ldots \ldots \ldots \ldots \ldots \ldots \ldots \ldots \ldots \ldots \ldots$

7. Ziele und Strategien . . . . . . . . . . . . . . . . . . . . . . 124

7.1 Ziele . . . . . . . . . . . . . . . . . . . . . . 124

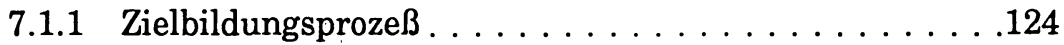

7.1.2 Zielsystem der Unternehmung . . . . . . . . . . . . . . 126

7.1.3 Empirische Befunde . . . . . . . . . . . . . . 126

7.2 Strategie . . . . . . . . . . . . . . . . . . . 130

7.2.1 Strategiebegriff. . . . . . . . . . . . . . . . 130

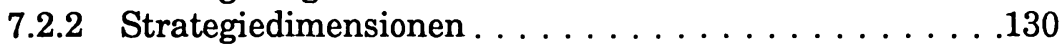

7.2.3 Strategische Planung . . . . . . . . . . . . . . . . . 132

7.3 Controlling . . . . . . . . . . . . . . . . . . . . 134

7.4 Theoretische Grundlagen . . . . . . . . . . . . . . . 135

8. Personalwirtschaft . . . . . . . . . . . . . . . . . . . . . 137

8.1 Begriff und Aufgabenstellung . . . . . . . . . . . . 137

8.2 Personalwirtschaftliche Gestaltungsfelder . . . . . . . . . . . . 139

8.2.1 Personalbereitstellung . . . . . . . . . . . . . . . 139

8.2.1.1 Personalbedarf. . . . . . . . . . . . . 139

8.2.1.2 Externe Personalbeschaffung . . . . . . . . . 140

8.2.1.3 Interne Personalbeschaffung . . . . . . . . . . . 142

8.2.2 Betriebliche Bildungsarbeit . . . . . . . . . . . . .146

8.2.2.1 Berufsausbildung . . . . . . . . . . . . . 147

8.2.2.2 Weiterbildung. . . . . . . . . . . . . . . . . . . 148

8.2 .3 Anreizgestaltung . . . . . . . . . . . . . . . . . 152

8.2 .4 Führung . . . . . . . . . . . . . . . . . . . . . . .154

8.2.4.1 Einheitliches zielgerichtetes Handeln . . . . . . .154

8.2.4.2 Rahmenbedingungen des Führungsgeschehens 154

8.2.4.3 Führungsverhalten und Führungsstile . . . . . 157 
8.2.4.4 Führungseigenschaften . . . . . . . . . . . 160

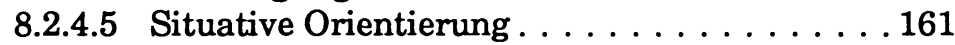

8.2.4.6 Führungsmodelle . . . . . . . . . . . 162

8.3. Theoretische Grundlagen . . . . . . . . . . . . . . . . 164

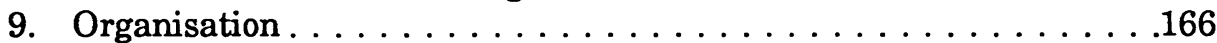

9.1 Grundlegung . . . . . . . . . . . . . . . . . . . 166

9.1.1 Der Organisationsbegriff . . . . . . . . . . . . . 166

9.1.2 Ziele des Organisierens . . . . . . . . . . . . . . 167

9.2 Organisatorische Gestaltungsfelder . . . . . . . . . . . 168

9.2.1 Die Aufgabengliederung . . . . . . . . . . . . . . 168

9.2.2 Leitungsstruktur . . . . . . . . . . . . . . . . . 174

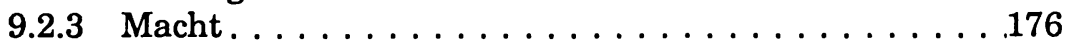

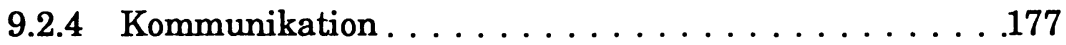

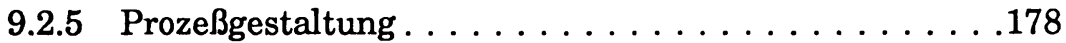

9.3 Organisationsstruktur und Organisationskontext . . . . . . 178

9.4 Organisatorischer Wandel . . . . . . . . . . . . . . . . . . 179

9.4.1 Merkmale von Prozessen des organisatorischen

Wandels . . . . . . . . . . . . . . . . . . . 179

9.4.2 Konzepte des organisatorischen Wandels . . . . . . . . 180

9.4.3 Phasen der Entwicklung von Organisationen . . . . . . . 182

9.5 Theoretische Grundlagen . . . . . . . . . . . . . . . 183

10. Internationale Unternehmenstätigkeit . . . . . . . . . . . . . . 185

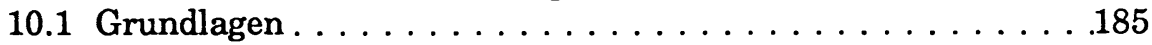

10.1.1 Definitionen. . . . . . . . . . . . . . . . 185

10.1.2 Ziele . . . . . . . . . . . . . . . . . . . 186

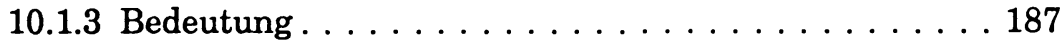

10.2 Merkmale des Auslandsgeschäfts . . . . . . . . . . . . . . . 189

10.3 Ausgewählte Gestaltungsfelder . . . . . . . . . . . . . . . 191

10.3.1 Export-Marketing . . . . . . . . . . . . . . . 191

10.3.2 Außenhandels-Logistik . . . . . . . . . . . . . . . . . 192

10.3.3 Außenhandels-Finanzierung . . . . . . . . . . . . . . 193

10.3.4 Internationale Personalarbeit . . . . . . . . . . 195

10.4 Theoretische Grundlagen . . . . . . . . . . . . . . . . 197

Teil 4: Werkzeuge $\ldots \ldots \ldots \ldots \ldots \ldots \ldots \ldots \ldots \ldots \ldots \ldots$

11. Rechnungswesen . . . . . . . . . . . . . . . . . . . .201

11.1 Grundlagen. . . . . . . . . . . . . . . . 201

11.1.1 Aufgaben und Ziele des Rechnungswesens . . . . . . . . 201

11.1.2 Grundbegriffe . . . . . . . . . . . . . . . 203

11.1.3 Teilgebiete des Rechnungswesens . . . . . . . . . . 206

11.2 Jahresabschluß . . . . . . . . . . . . . . . . . . .207

11.2.1 Bilanz . . . . . . . . . . . . . . . . . . . . . . 208

11.2.2 Gewinn- und Verlustrechnung . . . . . . . . . . . . .213

11.2.3 Weitere Bestandteile . . . . . . . . . . . . . . . . . . 214 
11.3 Kostenrechnung. . . . . . . . . . . . . . . . 215

11.3.1 Aufbau der Kostenrechnung . . . . . . . . . . . . 215

11.3.2 Hauptbestandteile der Kostenrechnung . . . . . . . . 217

11.3.3 Weiterentwicklungen der Kostenrechnung . . . . . . . 221

11.4 Investitionsrechnung . . . . . . . . . . . . . 222

11.4.1 Statische Verfahren . . . . . . . . . . . . . .223

11.4.2 Dynamische Verfahren . . . . . . . . . . . . . .223

11.5 Theoretische Grundlagen . . . . . . . . . . . . . . . . . 225

12. Computergestützte Informations- und

Kommunikationssysteme . . . . . . . . . . . . . . . . . . .228

12.1 Grundlagen . . . . . . . . . . . . . . . . . . 228

12.1.1 Gegenstand und begriffliche Grundlegung . . . . . . . .228

12.1.2 Bedeutung . . . . . . . . . . . . . . . . . . 228

12.2 Teilbereiche . . . . . . . . . . . . . . . . . . 229

12.2.1 Hardware . . . . . . . . . . . . . . . . . . . . .229

12.2.2 Software . . . . . . . . . . . . . . . . .234

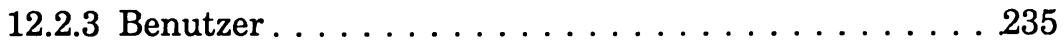

12.2.4 Aufgaben bzw. Anwendungen . . . . . . . . . . . . 235

12.3 Theoretische Grundlagen . . . . . . . . . . . . . . . . 236

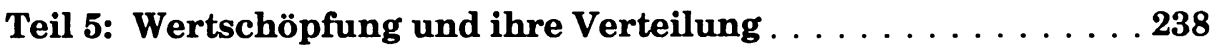

13. Lohn und Erfolgsbeteiligung . . . . . . . . . . . . . . 238

13.1 Wertschöpfung und Verteilungsgerechtigkeit . . . . . . . . 238

13.2 Anforderungsgerechtigkeit: Arbeitsbewertung . . . . . . . 241

13.3 Leistungsgerechtigkeit: Die Lohnformen . . . . . . . . . . . . .243

13.3.1 Akkordlohn . . . . . . . . . . . . . . . . .243

13.3.2 Prämienlohn . . . . . . . . . . . . . . . . .245

13.3.3 Zeitlohn . . . . . . . . . . . . . . . . . . . . 246

13.3.4 Automation und Lohnformen . . . . . . . . . . . . . .246

13.4 Erfolgsbeteiligung . . . . . . . . . . . . . . . . . . . 247

13.4.1 Ziele . . . . . . . . . . . . . . . . . . . 248

13.4.2 Formen der Mitarbeiterbeteiligung . . . . . . . . . 249

13.4.3 Ausgestaltung der Mitarbeiter-Erfolgsbeteiligung . . . 250

13.4.4 Kapitalbeteiligungen . . . . . . . . . . . . . . 252

13.5 Theoretische Grundlagen . . . . . . . . . . . . . . . . 253

Stichwortverzeichnis . . . . . . . . . . . . . . . .256 


\section{Abbildungsverzeichnis}

Abb. 1: Wissenschaftsbegriffe .................4

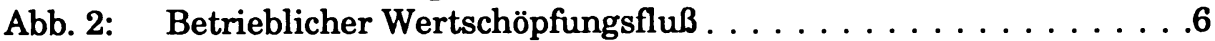

Abb. 3: Leistungswirtschaftliche Funktionen bzw. Teilprozesse . . . . . .8

Abb. 4: Managementbegriffe . . . . . . . . . . . . . .9

Abb. 5: Übersicht über die speziellen Betriebswirtschaftslehren . . . . .13

Abb. 6: Die Kernbereiche der betriebswirtschaftlichen Funktionslehren als Teil der Allgemeinen Betriebswirtschaftslehre . . . . . . . 16

Abb. 7: Das ökonomische Teilsystem im gesellschaftlichen Kontext . . 19

Abb. 8: Bruttosozialprodukt zu Marktpreisen in der Bundesrepublik Deutschland - Veränderungen gegenüber dem Vorjahr in \% . . . 23

Abb. 9: Betrieblich relevante Steuern . . . . . . . . . . . . .24

Abb. 10: Einflußfaktoren von Standortentscheidungen . . . . . . . . 26

Abb. 11: Rechtsformen des Privatrechts . . . . . . . . . . . . . . . .29

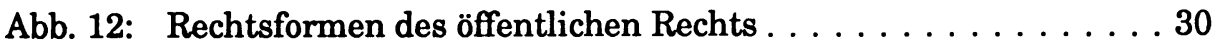

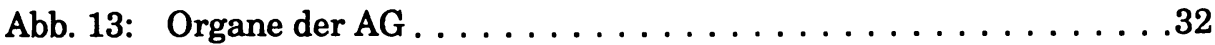

Abb. 14: Aufbau einer $\mathrm{GmbH} \&$ Co. KG . . . . . . . . . . . . . 33

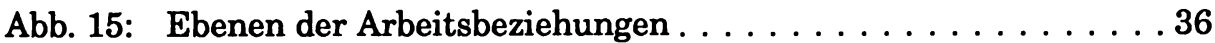

Abb. 16: Mitbestimmung auf Unternehmensebene . . . . . . . . .41

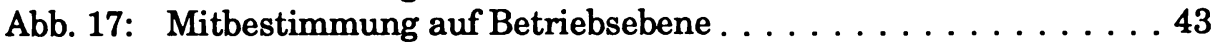

Abb. 18: Aufbau einer Arbeitnehmervertretung . . . . . . . . . . . .44

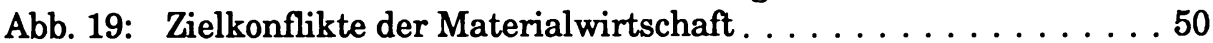

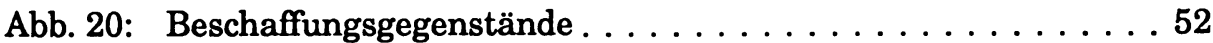

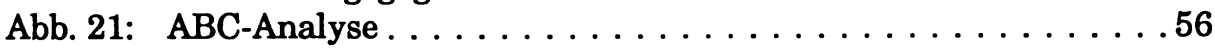

Abb. 22: Programmorientierte Materialbedarfsermittlung . . . . . . .57

Abb. 23: Bestellpunktverfahren . . . . . . . . . . . . . . . . .59

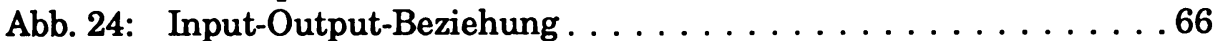

Abb. 25: Produktionsziele . . . . . . . . . . . . . . . . . .68

Abb. 26: Prozeßbetrachtung (Beschaffungs-, Produktions-

und Absatzlogistik) . . . . . . . . . . . . . 73

Abb. 27: Produktorientierte Perspektive der Produktion . . . . . . . . .73

Abb. 28: Konzept der computerintegrierten Fertigung (CIM) . . . . . . .78

Abb. 29: Gegenstand der Produktions- und Kostentheorie . . . . . . . . . 82

Abb. 30: Der absatzwirtschaftliche Prozeß . . . . . . . . . . . . . . 86

Abb. 31: Demographische Struktur in der Bundesrepublik Deutschland . 88

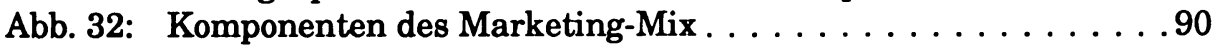

Abb. 33: Lebenszyklus eines Produktes . . . . . . . . . . . . . . .92

Abb. 34: Absatzwege . . . . . . . . . . . . . . . . . . .994

Abb. 35: Ausgleich der Finanzströme als Aufgabe der

Finanzwirtschaft des Unternehmens . . . . . . . . . . . . . . 104

Abb. 36: Kassenüberschuß oder Cash Flow . . . . . . . . . . . . . 105

Abb. 37: Kapitalbedarfsermittlung . . . . . . . . . . . . . . . 109

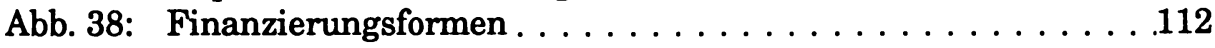


Abb. 39: Grundschema der Finanzplanung . . . . . . . . . . . . 114

Abb. 40: Finanzierungsregeln . . . . . . . . . . . . . . . 114

Abb. 41: Hauptaufgabenfelder des Managements . . . . . . . . . . 122

Abb. 42: Politisches System der Unternehmung . . . . . . . . . . . . . . . 124

Abb. 43: Ziel- und Planungsebenen . . . . . . . . . . . . . . . 132

Abb. 44: Marktanteil - Marktwachstum - Portfolio . . . . . . . . . . . . 134

Abb. 45: Kernaufgaben der Personalwirtschaft . . . . . . . . . . 138

Abb. 46: Elemente von Personalentwicklungssystemen . . . . . . . . . 146

Abb. 47: Betriebliche Weiterbildungsintensität . . . . . . . . . . . 149

Abb. 48: Sicherung einheitlichen zielgerichteten Handelns

durch Führung . . . . . . . . . . . . . . . 155

Abb. 49: Varianten des Führungsstils . . . . . . . . . . . . . . . 159

Abb. 50: Kompetenzen erfolgreicher Manager . . . . . . . . . . . 161

Abb. 51: Idealtypische Führungsformen . . . . . . . . . . . 163

Abb. 52: Beispiel für die Funktionalorganisation . . . . . . . . . . . . 170

Abb. 53: Divisionalisierung . . . . . . . . . . . . . . . . . . . 171

Abb. 54: Matrix-Organisation (produkt- und funktionsorientiert) . . . . 172

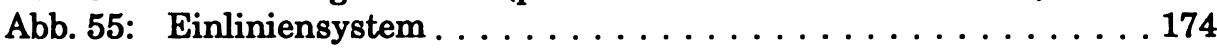

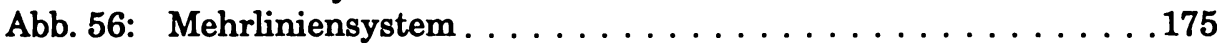

Abb. 57: Stabliniensystem . . . . . . . . . . . . . . . . . . 175

Abb. 58: Merkmale und Konzepte des organisatorischen Wandels . . . 181

Abb. 59: Stufen der internationalen Unternehmenstätigkeit . . . . . . . 185

Abb. 60: Warenstruktur des Außenhandels der Bundesrepublik (1991) .188

Abb. 61: Chancen und Probleme des Auslandsgeschäftes . . . . . . . . . 191

Abb. 62: Ablauf eines Akkreditiv-Geschäfts . . . . . . . . . . . . . . . . . . 194

Abb. 63: Abgrenzung von Aufwand und Kosten . . . . . . . . . . . . 205

Abb. 64: Abgrenzung von Ertrag und Leistung . . . . . . . . . . . . . 206

Abb. 65: Teilgebiete des Rechnungswesens . . . . . . . . . . . . 206

Abb. 66: Aufbau einer Bilanz . . . . . . . . . . . . . . . . . 208

Abb. 67: Bilanzgliederung nach \& 266 HGB für eine

Kapitalgesellschaft (komprimierte Fassung) . . . . . . . . . . 212

Abb. 68: Aufbau der Kostenrechnung . . . . . . . . . . . . . . . . .216

Abb. 69: Grobschema eines Kostenartenplans . . . . . . . . . . . 217

Abb. 72: Anteil von Berufen mit EDV-Kenntnissen . . . . . . . . . . 229

Abb. 73: Hauptelemente eines Computers . . . . . . . . . . . . . .231

Abb. 74: Die Stellung der Wirtschaftsinformatik . . . . . . . . . . . . 236

Abb. 75: Kriterien und Instrumente der Lohnfindung . . . . . . . . . . . .240

Abb. 76: Grundtypen der Arbeitsbewertung . . . . . . . . . . 241

Abb. 77: Genfer Schema . . . . . . . . . . . . . . . . . . . .242

Abb. 78: Prämienarten . . . . . . . . . . . . . . . . . . . .246

Abb. 79: Mögliche Lohnformen bei verschiedenen Stufen der

Mechanisierung und Automation . . . . . . . . . 247

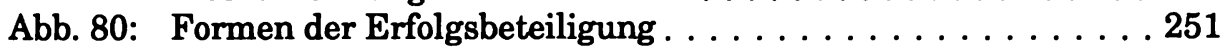




\section{Tabellenverzeichnis}

Tab. 1 Unternehmen und Beschäftigte nach Rechtsformen . . . . . . . .34

Tab. 2 Anteil des Materialverbrauchs einschließlich Energieverbrauch am Bruttoproduktionswert ausgewählter

Wirtschaftszweige . . . . . . . . . . . . . . . . .51

Tab. 3 Nettoeinnahmen der Werbeträger (Medien) in der Bundesrepublik Deutschland . . . . . . . . . . . . . . . .96

Tab. 4 Eigenkapitalquote der Industrie (Aktiengesellschaften des produzierenden Gewerbes in der Bundesrepublik Deutschland). . . . . . . . . . . . . . . . . . 115

Tab. 5 Eigenkapitalquote ausgewählter Gewerbezweige . . . . . . . . . .117

Tab. 6 Ausfuhr der Bundesrepublik Deutschland 1991 nach Ländergruppen . . . . . . . . . . . . . . . . . . . . . . . . . 189

Tab. 7 Grundbegriffe des Rechnungswesens . . . . . . . . . . . 204 